\title{
Model Reduction in Kinetic Theory
}

\author{
Henning Struchtrup \\ Department of Mechanical Engineering, University of Victoria, PO Box STN CSC \\ 3055, Victoria BC V8W 3P6, Canada, struchtr@me.uvic.ca
}

Summary. Methods to derive macroscopic transport equations for rarefied gases from the Boltzmann equations are presented. Featured methods include the Chapman-Enskog expansion, Grad's moment method, and the author's order of magnitude method. The resulting macroscopic equations are compared and discussed by means of simple problems, including linear stability, shock wave structures, and Couette flow.

\section{Introduction}

The most important scaling parameter to characterize processes in rarefied gases is the Knudsen number $\mathrm{Kn}$, defined as the ratio between the mean free path of a particle and a relevant reference length scale (e.g. channel width, wavelength, etc.). For a complex flow the local Knudsen number might differ by several orders of magnitude between locations. Thus, rarefied gas flows are multiscale problems.

Processes in rarefied gases are well described by the Boltzmann equation $[1,2,3]$, a non-linear integro-differential equation that describes the evolution of the particle distribution function $f$ in phase space, i.e. on the microscopic level. The numerical solution of the Boltzmann equation, either directly [4] or via the Direct Simulation Monte Carlo (DSMC) method [5], is very time expensive.

If the Knudsen number is small, the Boltzmann equation can be reduced to simpler models, which allow faster solutions,

If $\mathrm{Kn}<0.01$ (say), the equations of ordinary hydrodynamics - the laws of NavierStokes and Fourier (NSF) — can be derived from the Boltzmann equation. The NSF equations are macroscopic equations for mass density $\rho$, velocity $v_{i}$ and temperature $T$, and thus pose a mathematically less complex problem than the Boltzmann equation.

Macroscopic equations for rarefied gas flows at Knudsen numbers above 0.01 are highly desirable, since they promise to replace the Boltzmann equation with simpler equations that still capture the relevant physics. Several methods are available to derive the desired higher order equations, and all of these suggest different sets of equations. 
Naturally, the complexity of the subject forbids a detailed discussion of this rather complex topic on the space available. Thus, this contribution aims mainly at presenting the main ideas of the most important methods, and to point out the relations and differences between the various sets of equations. The reader searching for greater detail is referred to the cited literature, in particular to the author's textbook [3].

\section{Basic Kinetic Theory}

We shall consider mon-atomic ideal gases exclusively. The basic quantity in kinetic theory is the particle distribution function $f(\mathbf{x}, t, \mathbf{c}) ; \mathbf{x}$ and $t$ are the space and time variables, respectively, and $\mathbf{c}$ denotes the microscopic velocities of particles. The distribution function is defined such that $f(\mathbf{x}, t, \mathbf{c}) d \mathbf{c} d \mathbf{x}$ gives the number of gas particles in the phase space cell $d \mathbf{c} d \mathbf{x}$ at time $t$.

Macroscopic quantities are obtained by taking suitable averages (moments) of the phase density. The basic hydrodynamic variables are obtained according to

$$
\rho=m \int f d \mathbf{c}, \rho v_{i}=m \int c_{i} f d \mathbf{c}, \rho u=\frac{3}{2} \rho \theta=\frac{m}{2} \int C^{2} f d \mathbf{c} .
$$

Here, $\theta=\frac{k}{m} T$ is the temperature in energy units (that will be used from now on instead of $T$ ), $m$ is the mass of a particle, $k$ denotes Boltzmann's constant, and $C_{i}=c_{i}-v_{i}$ is the peculiar velocity. $u$ denotes the specific internal energy, and $(1)_{3}$ must be considered as the definition of temperature.

The phase density is obtained as the solution of the Boltzmann equation,

$$
\frac{\partial f}{\partial t}+c_{i} \frac{\partial f}{\partial x_{i}}=\mathcal{S}(f, f)
$$

Here, $\mathcal{S}$ is the collision term which describes the change of $f$ due to collisions among particles. The full expression for $\mathcal{S}$ can be found in the literature $[1,2,3]$, here we only list its most important properties:

1. Mass, momentum and energy are conserved in a collision,

$$
m \int \mathcal{S} d \mathbf{c}=0, m \int c_{i} \mathcal{S} d \mathbf{c}=0, \frac{m}{2} \int C^{2} \mathcal{S} d \mathbf{c}=0 .
$$

2. The production of entropy is always non-negative (H-theorem),

$$
\Sigma=-k \int \ln f \mathcal{S} d \mathbf{c} \geqslant 0
$$

3. In equilibrium the phase density is a Maxwellian distribution, i.e.

$$
\mathcal{S}=0 \Longrightarrow f=f_{M}=\frac{\rho}{m} \frac{1}{\sqrt{2 \pi \theta}^{3}} \exp \left[-\frac{C^{2}}{2 \theta}\right] \text {. }
$$

The Boltzmann collision term $\mathcal{S}$ is a complex non-linear integral expression in $f$ that depends also on the interaction potential between the particles. Its mathematical treatment becomes particularly simple for particles interacting with a repulsive 
fifth-order power potential, the so-called Maxwell molecules. More realistic potentials, e.g. general power laws, hard sphere molecules, or Lennard-Jones potentials introduce higher complexity.

Simplified expressions for $\mathcal{S}$ that capture its basic properties are often used, the most popular of these is the BGK model [6] where

$$
\mathcal{S}_{B G K}=\nu\left(f_{M}-f\right) ;
$$

$\nu$ is the average collision frequency for a particle.

Multiplication of the Boltzmann equation (2) with $\left\{m, m c_{i}, \frac{m}{2} C^{2}\right\}$ and subsequent integration over the microscopic velocity yields the conservation laws for mass, momentum and internal energy,

$$
\begin{aligned}
\frac{\partial \rho}{\partial t}+\frac{\partial \rho v_{k}}{\partial x_{k}} & =0 \\
\rho \frac{\partial v_{i}}{\partial t}+\rho v_{k} \frac{\partial v_{i}}{\partial x_{k}}+\frac{\partial p}{\partial x_{i}}+\frac{\partial \sigma_{i k}}{\partial x_{k}} & =0 \\
\frac{3}{2} \rho \frac{\partial \theta}{\partial t}+\frac{3}{2} \rho v_{k} \frac{\partial \theta}{\partial x_{k}}+\frac{\partial q_{k}}{\partial x_{k}} & =-\left(p \delta_{i j}+\sigma_{i j}\right) \frac{\partial v_{i}}{\partial x_{j}} .
\end{aligned}
$$

Here, the pressure $p$ obeys the ideal gas law, $p=\rho \theta$, and

$$
q_{i}=\frac{m}{2} \int C^{2} C_{i} f d \mathbf{c}
$$

denotes the heat flux vector. The pressure tensor is defined as

$$
p \delta_{i j}+\sigma_{i j}=m \int C_{i} C_{j} f d \mathbf{c} \quad \text { with } \quad \sigma_{i j}=m \int C_{\langle i} C_{j\rangle} f d \mathbf{c}
$$

where $\sigma_{i j}$ denotes the stress, that is the symmetric and tracefree part of the pressure tenor, with $\sigma_{i i}=0, \sigma_{i j}=\sigma_{j i}$. Indices in angular brackets denote symmetric tracefree tensors (see [3], Appendix A.2).

The conservation laws (7) together with the definitions for stress and heat flux $(9,8)$ are exact, that is they are valid for any solution $f$ of the Boltzmann equation. Mathematically, the five conservation laws do not form a closed set of equations for the hydrodynamic variables $\left\{\rho, v_{i}, \theta\right\}$, since they contain stress and heat flux as well.

The idea of macroscopic continuum approximations is to close the set of equations by deriving additional macroscopic equations for $\sigma_{i j}$ and $q_{i}$ from the Boltzmann equation by means of rational approximation procedures. Various methods available to this end, and the corresponding additional equations for $\sigma_{i j}$ and $q_{i}$, will be discussed in the sequel.

For completeness we mention that multiplication of the Boltzmann equation with $-k \ln \frac{f}{y}$ ( $y$ is a constant) yields the balance of entropy, which has a non-negative production (4) $[1,2,3]$.

\section{Chapman-Enskog method}

The best known approach to derive macroscopic transport equations from the Boltzmann equation is the Chapman-Enskog (CE) method $[1,2,3,7,8]$. The CE method 
is based on the dimensionless form of the Boltzmann equation which contains the Knudsen number as a scaling parameter for the collision term,

$$
\frac{\partial f}{\partial t}+c_{i} \frac{\partial f}{\partial x_{i}}=\frac{1}{\mathrm{Kn}} \mathcal{S}(f, f)
$$

In the limit $\mathrm{Kn} \rightarrow 0$, the collision term must vanish, and it follows from the properties of $\mathcal{S}$ that the corresponding phase density is the local Maxwellian $(5), f^{(0)}=f_{M}$. Evaluation of $\sigma_{i j}$ and $q_{i}$ with the Maxwellian gives zero stress and heat flux,

$$
\sigma_{i j}^{(0)}=q_{i}^{(0)}=0
$$

Insertion of this into the conservation laws (7) yields the well known Euler equations.

The idea of the CE expansion method is to add corrections to the local equilibrium distribution by adding terms of higher orders in the Knudsen number,

$$
f=f^{(0)}+\mathrm{Kn} f^{(1)}+\mathrm{Kn}^{2} f^{(2)}+\cdots .
$$

An important condition on the expansion (12) is that the hydrodynamic variables $\left\{\rho, v_{i}, \theta\right\}$ are the same at any level of expansion, so that

$$
\rho\left\{1, v_{i}, \frac{3}{2} \theta\right\}=m \int\left\{1, c_{i}, \frac{C^{2}}{2}\right\} f^{(0)} d \mathbf{c}, 0=\int\left\{1, c_{i}, \frac{C^{2}}{2}\right\} f^{(\alpha)} d \mathbf{c}(\alpha \geq 1) .
$$

These compatibility conditions guarantee that only the equations for the nonequilibrium variables $\sigma_{i j}$ and $q_{i}$ change with increasing degree of approximation,

$$
\sigma_{i j}=\sigma_{i j}^{(0)}+\mathrm{Kn} \sigma_{i j}^{(1)}+\mathrm{Kn}^{2} \sigma_{i j}^{(2)}+\cdots, \quad q_{i}=q_{i}^{(0)}+\mathrm{Kn} q_{i}^{(1)}+\mathrm{Kn}^{2} q_{i}^{(2)}+\cdots .
$$

The expansion parameters $f^{(\alpha)}$ are determined successively by plugging the series (12) into the Boltzmann equation, and equating terms with the same factors in powers of the Knudsen number. This leads to an iterative structure, where the correction at order $\alpha$ is a function of (derivatives of) the lower order corrections, $f^{(\alpha)}=\mathcal{F}\left(f^{(\beta)}, 0 \leq \beta<\alpha\right)$, see e.g. [7, 3]. All correction terms depend only on the hydrodynamic variables and their gradients, ${ }^{\dagger}$ since the zeroth order term-the Maxwellian - depends only on the hydrodynamic variables $\left\{\rho, v_{i}, \theta\right\}$. Stress and heat flux are computed from the approximation (12) by accounting for terms up to a certain order, and the resulting expressions will relate $\sigma_{i j}$ and $q_{i}$ to the hydrodynamic variables and their gradients.

Obviously, to zeroth order the expansion yields the Euler equations (11). The first order correction gives the laws of Navier-Stokes and Fourier,

$$
\sigma_{i j}^{(1)}=-2 \mu \frac{\partial v_{\langle i}}{\partial v_{j\rangle}} \quad, \quad q_{i}^{(1)}=-\kappa \frac{\partial \theta}{\partial x_{i}} .
$$

The most important success of the CE method is that it gives accurate expressions for viscosity $\mu$ and heat conductivity $\kappa$, which relate these to the microscopic interaction potential and the hydrodynamic variables. In particular one finds, in accordance with experiments, that the viscosity depends only on temperature, and not on density. For power potentials the CE method yields

\footnotetext{
$\dagger$ Time derivatives are replaced by means of the conservation laws $[7,3]$.
} 
\begin{tabular}{|l|l||l|l|l|l|l|l|l|l|l|l|l|}
\hline$\gamma$ & $\omega$ & $\varpi_{1}$ & $\varpi_{2}$ & $\varpi_{3}$ & $\varpi_{4}$ & $\varpi_{5}$ & $\varpi_{6}$ & $\theta_{1}$ & $\theta_{2}$ & $\theta_{3}$ & $\theta_{4}$ & $\theta_{5}$ \\
\hline
\end{tabular}

\begin{tabular}{|l||l|l|l|l|l|l|l|l|l|l|l|}
\hline & $10 / 3$ & 2 & 3 & 0 & 3 & 8 & $75 / 8$ & $45 / 8$ & -3 & 3 & $39 / 4$ \\
\hline \hline 5 & 1 & $10 / 6$ & 0.8 & 3.600 & 2.004 & 2.761 & 0.254 & 1.784 & 7.748 & 100 & \\
\hline
\end{tabular}

\begin{tabular}{|l|l||l|l|l|l|l|l|l|l|l|l|l|}
\hline 7.66 & 0.8 & 3.600 & 2.004 & 2.761 & 0.254 & 1.784 & 7.748 & 10.160 & 5.656 & -3.014 & 2.761 & 9.019 \\
\hline
\end{tabular}

\begin{tabular}{|l|l||l|l|l|l|l|l|l|l|l|l|l|}
\hline$\infty$ & 0.5 & 4.056 & 2.028 & 2.418 & 0.681 & 0.219 & 7.424 & 11.644 & 5.822 & -3.09 & 2.418 & 8.286 \\
\hline
\end{tabular}

Table 1: Burnett coefficients for power potentials $(\gamma=5$ for Maxwell molecules, $\gamma=7.66$ for argon, $\gamma=\infty$ for for hard sphere molecules) [10].

$$
\mu=\mu_{0}\left(\frac{\theta}{\theta_{0}}\right)^{\omega}
$$

with $\omega=1 / 2$ for hard spheres and $\omega=1$ for Maxwell molecules; experiments indicate $\omega \simeq 0.8$ for argon [5]. Heat conductivity and viscosity are related through the Prandtl number, ${ }^{\dagger}$

$$
\operatorname{Pr}=\frac{5}{2} \frac{\mu}{\kappa} \simeq \frac{2}{3}
$$

The value of Pr varies only slightly (less than $1 \%$ ) with the molecule model, and measured values are close to $0.66[2,3]$.

The second order contributions are the Burnett equations $[9,2,7,3]$,

$$
\begin{array}{r}
\sigma_{i j}^{(2)}=\frac{\mu^{2}}{p}\left[\varpi_{1} \frac{\partial v_{k}}{\partial x_{k}} S_{i j}-\varpi_{2}\left(\frac{\partial}{\partial x_{\langle i}}\left(\frac{1}{\rho} \frac{\partial p}{\partial x_{j\rangle}}\right)+\frac{\partial v_{k}}{\partial x_{\langle i}} \frac{\partial v_{j\rangle}}{\partial x_{k}}+2 \frac{\partial v_{k}}{\partial x_{\langle i}} S_{j\rangle k}\right)\right. \\
\left.+\varpi_{3} \frac{\partial^{2} \theta}{\partial x_{\langle i} \partial x_{j\rangle}}+\varpi_{4} \frac{\partial \theta}{\partial x_{\langle i}} \frac{\partial \ln p}{\partial x_{j\rangle}}+\varpi_{5} \frac{1}{\theta} \frac{\partial \theta}{\partial x_{\langle i}} \frac{\partial \theta}{\partial x_{j\rangle}}+\varpi_{6} S_{k\langle i} S_{j\rangle k}\right], \\
q_{i}^{(2)}=\frac{\mu^{2}}{\rho}\left[\theta_{1} \frac{\partial v_{k}}{\partial x_{k}} \frac{\partial \ln \theta}{\partial x_{i}}-\theta_{2}\left(\frac{2}{3} \frac{\partial^{2} v_{k}}{\partial x_{k} \partial x_{i}}+\frac{2}{3} \frac{\partial v_{k}}{\partial x_{k}} \frac{\partial \ln \theta}{\partial x_{i}}+2 \frac{\partial v_{k}}{\partial x_{i}} \frac{\partial \ln \theta}{\partial x_{k}}\right)\right. \\
\left.+\theta_{3} S_{i k} \frac{\partial \ln p}{\partial x_{k}}+\theta_{4} \frac{\partial S_{i k}}{\partial x_{k}}+3 \theta_{5} S_{i k} \frac{\partial \ln \theta}{\partial x_{k}}\right] .
\end{array}
$$

The Burnett coefficients $\varpi_{\alpha}, \theta_{\alpha}$ depend on the molecule type, and for power potentials with exponent $\gamma$ some values are given in Table 1 [10].

The third order expansion yields the super-Burnett equations. Their computation is extremely cumbersome, and the full three-dimensional non-linear superBurnett equations were never derived. One only finds the linearized equations in $3-\mathrm{D}[11,12,13,3]$, and the non-linear equations for one-dimensional geometry $[11,14,15,3]$.

The equations of Navier-Stokes and Fourier cease to be accurate for Knudsen numbers above $\sim 0.01$, and one would expect that Burnett and super-Burnett equations are valid for larger Knudsen numbers. Unfortunately, however, the higher order equations become linearly unstable for processes involving small wavelengths, or high frequencies $[12,15]$, and they lead to unphysical oscillations in steady state processes [16], and thus cannot be used in numerical simulations .

There is no clear argument why the Chapman-Enskog expansion leads to unstable equations. It seems that a first order Chapman-Enskog expansion leads generally

\footnotetext{
$\dagger$ Our defintion of the Prandtl number differs from the usual one by a factor $\frac{k}{m}$ due to the use of $\theta$ instead of $T$.
} 
to stable equations, while higher order expansions generally yield unstable equations, although exceptions apply, e.g. see [17, 18].

Zhong et al. suggested the "augmented Burnett equations" where some terms of super-Burnett order (but not the actual super-Burnett terms) are added to the Burnett equations to stabilize these $[19,20]$. The augmented Burnett equations still are unstable in space $[15,3]$, and they lack a rational derivation from the Boltzmann equation [15].

For reference in subsequent sections we print the distribution function for the NSF equations (with $\xi=C / \sqrt{\theta}$ )

$$
f_{\mid C E}=f_{M}\left[1+\frac{\sigma_{i k}^{(1)}}{2 p} A\left(\xi^{2}\right) \frac{C_{\langle i} C_{k\rangle}}{\theta}+\frac{2}{5} \frac{q_{k}^{(1)}}{p \theta} B\left(\xi^{2}\right) C_{k}\left(\frac{C^{2}}{2 \theta}-\frac{5}{2}\right)\right] .
$$

The dimensionless functions $A$ and $B$ result from the approximate inversion of the Boltzmann collision term $[2,7,3]$ and thus depend on the interaction potential. For Maxwell molecules they are constants, $A=B=1$, while for all other interaction potentials they are polynomials; fourth order polynomials give an excellent approximation $[2,10]$. Setting $A=B=1$ leads to small deviations between theory and measurement of viscosity and heat conductivity.

\section{Grad moment method}

The Chapman-Enskog method leads to expressions for stress and heat flux that contain higher derivatives of the hydrodynamic variables. Grad suggested a quite different approach, in which the number of variables is extended beyond the 5 hydrodynamic variables $\rho, v_{i}, \theta$, by adding stress $\sigma_{i j}$, heat flux $q_{i}$ and other moments to the list of variables $[21,22]$. The corresponding transport equations for the additional variables are obtained as moments of the Boltzmann equation and are first order partial differential equations for the moments. They do not form a closed set for the variables and require a closure argument. For this Grad suggests to approximate the phase density by an expansion in Hermite polynomials about the equilibrium distribution (the local Maxwellian), where the expansion coefficients are related to the moments.

A crucial point in the method is the question which and how many moments are needed to describe a process. The answer depends on the particular process, but experience shows that the number of moments must be increased with increasing Knudsen number [23, 24, 25, 26, 27, 28]. Grad's method does not provide an argument that links the Knudsen number to the set of moments to be considered as variables.

The best known set of Grad-type moment equations is the 13 moment system, which has the variables $\left\{\rho, v_{i}, \theta, \sigma_{i j}, q_{i}\right\}$. The corresponding moment equations are obtained by multiplying the Boltzmann equation with $\left\{m, m c_{i}, \frac{m}{2} C^{2}, m C_{\langle i} C_{j\rangle}, \frac{m}{2} C^{2} C_{i}\right\}$. This gives the conservation laws (7) plus additional moment equations for stress and heat flux,

$$
\frac{\partial \sigma_{i j}}{\partial t}+v_{k} \frac{\partial \sigma_{i j}}{\partial x_{k}}+\sigma_{i j} \frac{\partial v_{k}}{\partial x_{k}}+\frac{4}{5} \frac{\partial q_{\langle i}}{\partial x_{j\rangle}}+2 p \frac{\partial v_{\langle i}}{\partial x_{j\rangle}}+2 \sigma_{k\langle i} \frac{\partial v_{j\rangle}}{\partial x_{k}}+\frac{\partial m_{i j k}}{\partial x_{k}}=\mathcal{P}_{i j},
$$




$$
\begin{aligned}
\frac{\partial q_{i}}{\partial t}+v_{k} \frac{\partial q_{i}}{\partial x_{k}}+ & \frac{5}{2} p \frac{\partial \theta}{\partial x_{i}}+\frac{5}{2} \sigma_{i k} \frac{\partial \theta}{\partial x_{k}}+\theta \frac{\partial \sigma_{i k}}{\partial x_{k}}-\theta \sigma_{i k} \frac{\partial \ln \rho}{\partial x_{k}}+\frac{7}{5} q_{k} \frac{\partial v_{i}}{\partial x_{k}}+\frac{2}{5} q_{k} \frac{\partial v_{k}}{\partial x_{i}} \\
& +\frac{7}{5} q_{i} \frac{\partial v_{k}}{\partial x_{k}}+\frac{1}{2} \frac{\partial R_{i k}}{\partial x_{k}}+\frac{1}{6} \frac{\partial \Delta}{\partial x_{i}}+m_{i j k} \frac{\partial v_{j}}{\partial x_{k}}-\frac{\sigma_{i j}}{\rho} \frac{\partial \sigma_{j k}}{\partial x_{k}}=\mathcal{P}_{i}
\end{aligned}
$$

Equations $(19,20)$ contain additional moments of the distribution function, which are defined as

$$
\begin{aligned}
\Delta & =m \int C^{4}\left(f-f_{M}\right) d \mathbf{c}, R_{i j}=m \int\left(C^{2}-7 \theta\right) C_{\langle i} C_{j\rangle} f d \mathbf{c}, \\
m_{i j k} & =m \int C_{\langle i} C_{j} C_{k\rangle} f d \mathbf{c} .
\end{aligned}
$$

The terms on the right hand sides are the moments of the Boltzmann collision term,

$$
\mathcal{P}_{i j}=m \int C_{\langle i} C_{j\rangle} \mathcal{S} d \mathbf{c}, \quad \mathcal{P}_{i}=\frac{m}{2} \int C^{2} C_{i} \mathcal{S} d \mathbf{c} .
$$

Obviously, the set of equations can be closed by finding expressions for $\Delta, R_{i j}, m_{i j k}, \mathcal{P}_{i j}, \mathcal{P}_{i}$ that relate these to the basic 13 variables $\left\{\rho, v_{i}, \theta, \sigma_{i j}, q_{i}\right\}$. To this end, the Grad method provides the distribution [21, 22, 3]

$$
f_{\mid 13}=f_{M}\left[1+\frac{\sigma_{i k}}{2 p} \frac{C_{\langle i} C_{k\rangle}}{\theta}+\frac{2}{5} \frac{q_{k}}{p \theta} C_{k}\left(\frac{C^{2}}{2 \theta}-\frac{5}{2}\right)\right] .
$$

This function recovers the basic 13 variables, and allows to compute the unknowns $(21,22)$ as

$$
\Delta=R_{i j}=m_{i j k}=0, \mathcal{P}_{i j}=-\frac{p}{\mu} \sigma_{i j}, \mathcal{P}_{i}=-\frac{2}{3} \frac{p}{\mu} q_{i} .
$$

Insertion of $(23)$ into $(19,20)$ gives, together with $(7)$, the closed set of equations for the 13 variables.

By comparing the distribution functions (18) and (23) it becomes evident that they are quite similar. However, there are two differences: (a) the CE phase density contains only the first approximations to stress and heat flux, $\sigma_{i j}^{(1)}$ and $q_{i}^{(1)}$, while the Grad distribution contains both as independent variables, $\sigma_{i j}$ and $q_{i}$. (b) In the Grad function, the coefficients $A$ and $B$ assume the values for Maxwell molecules, $A=B=1$. From the last point one will infer that the Grad 13 moment equations will be best suited for Maxwell molecules, while for other molecule types they can only be an approximation.

The Grad 13 equations have two major drawbacks: (a) The equations are symmetric hyperbolic for most values of the variables, and this leads to shock structures with discontinuities (sub-shocks) for Mach numbers above 1.65 [23, 26]. (b) Since Grad's method is not linked to the Knudsen number, the range of applicability for the equations is unclear.

These problems remain for Grad-type equations with more variables, which give smooth shocks up to higher but not too high Mach numbers [26, 27]. The 13 moment equations do not describe Knudsen boundary layers [29, 30, 24], increasing the number of moments allows to compute these [31, 24, 32].

For some problems, in particular for large Mach or Knudsen numbers, one has to face hundreds of moment equations, but the relation between moment number and 
Knudsen or Mach number is not clear. Computations for hundreds of moments are only manageable for simple geometries and problems [33, 23, 24], and were never performed in two or three dimensions. Indeed, the goal of a macroscopic set of equations must be to have a simplification compared to the Boltzmann equation, and using hundreds of moments does not achieve this goal.

\section{Combining the Chapman-Enskog and Grad methods}

In most of the available literature, the two classical methods - Grad moment method and Chapman-Enskog expansion - are treated as being completely unrelated. However, using a method akin to the Maxwellian iteration of Truesdell and Ikenberry $[34,35]$, Reinecke and Kremer extract the Burnett equations from Grad-type moment systems $[10,36]$. Which set of moments one has to use for this purpose depends on the interaction potential. For Maxwell molecules it is sufficient to consider Grad's set of 13 moments.

In [30] it was shown that this iteration method is equivalent to the CE expansion of the moment equations. In the original $\mathrm{CE}$ method one first expands, and then integrates the resulting distribution function to compute its moments. In the Reinecke-Kremer-Grad method, the order of integration and expansion is exchanged.

For Maxwell molecules the Burnett equations result from the second order CE expansion of the 13 moments set, while the super-Burnett equations result from the 3rd order CE expansion of the 26 moment set (which adds $\Delta, R_{i j}, m_{i j k}$ to the list of variables) [30, 13, 15, 3].

While the Reinecke-Kremer-Grad method does not give new results, it allows an easier access to higher order CE expansions, in particular the Burnett equations. The method does not solve the stability problems of the Burnett equations

M. Torrilhon and the present author used a different way to combine the two methods by assuming different time scales for the 13 basic variables of the theory on one side, and all higher moments on the other $[13,15,3]$. This allows to perform a CE expansion around a non-equilibrium state which is defined through the 13 variables. This method, which appeared first as a side note in Grad's contribution to the Encyclopedia of Physics [22], gives a regularizing correction to the Grad13 equations, the regularized 13 moment equations (R13 Eqs.).

The same idea was used by Karlin et al. [37] for the linearized Boltzmann equation. They compute an approximation to the distribution function, which is used to derive a set of 13 linear equations for the 13 moments. Their equations are the linearized form of the R13 equations.

The R13 equations are not hyperbolic, give smooth shock structures for all Mach numbers, and they are stable. Therefore, this combination of the CE and Grad methods yields a marked improvement over the original methods. The R13 equations will be shown and discussed later, in Sec. 6, which presents an alternative method of derivation.

The Grad distributions, e.g. the 13 moment phase density $f_{\mid 13}(23)$, define nonequilibrium manifolds in phase space $[37,38]$. The Maxwellians form a subset on these non-equilibrium manifolds (they are the appropriate Grad distribution for the 5 moments case, i.e. the Euler equations). The Grad closure restricts the phase space so that the gas cannot access all states in phase space, but only those on the 
Grad non-equilibrium manifold. This strong restriction is inherent to Grad's closure, and has no physical foundation, since Grad distributions cannot be extracted from the Boltzmann equation. This is different, of course, for the Maxwellians, which are those phase densities that give a zero collision term. With no argument from physics to support the Grad distributions, it seems to be daring to restrict the gas on the Grad non-equilibrium manifolds. One way to relax the Grad assumption - at least somewhat - is to allow states in the vicinity of the Grad manifolds. This stands in analogy to the relation between Euler and NSF equations, which describe the equilibrium manifold, and its vicinity.

It must be emphasized that there is no evidence in physics to support the existence of pseudo-equilibrium manifolds for the gas. In particular there is no guideline for choosing the relevant moments, or the pseudo-equilibrium distribution, which could, e.g., be a Grad distribution with any number of moments. A somewhat popular alternative are distribution functions that result from maximizing entropy or extended thermodynamics, see [23, 39, 40, 41, 42, 43] as well as [3] for details on, and problems associated with, this approach.

\section{Order of Magnitude Method}

The weak point in the Grad method is that no statement is made to connect Knudsen numbers and relevant moments. As a result, the derivation of the R13 equations as outlined above required the assumption of different time scales for the basic 13 moments, and higher moments. While this assumption leads to a set of equations with desired behavior, it is difficult to justify, since the characteristic times of all moments are, in fact, of the same order.

An alternative approach to the problem was presented by Struchtrup in $[44,45$, 3], partly based on earlier work by Müller et al. [46].

The order of magnitude method considers not the Boltzmann equation itself, but its infinite system of moment equations for symmetric and trace-free moments

$$
u_{\left\langle i_{i} \cdots i_{n}\right\rangle}^{(a)}=m \int C^{2 a} C_{\left\langle i_{1}\right.} \cdots C_{\left.i_{n}\right\rangle} f d \mathbf{c} \quad(a, n=0,1,2, \ldots) .
$$

Here, due to space restrictions, we cannot present the method in detail, but only describe its main steps; in particular we shall not show the general moment equations for the moments (25).

The method of finding the proper equations with order of accuracy $\lambda_{0}$ in the Knudsen number consists of the following three steps:

1. Determination of the order of magnitude $\lambda$ of the moments.

2. Construction of a moment set with minimum number of moments at any order $\lambda$.

3. Deletion of all terms in all equations that would lead only to contributions of orders $\lambda>\lambda_{0}$ in the conservation laws for energy and momentum.

Step 1 is based on a Chapman-Enskog expansion where a moment $\phi$ is expanded according to

$$
\phi=\phi_{0}+\mathrm{Kn} \phi_{1}+\mathrm{Kn}^{2} \phi_{2}+\mathrm{Kn}^{3} \phi_{3}+\cdots
$$


and the leading order of $\phi$ is determined by inserting this ansatz into the complete set of moment equations. A moment is said to be of leading order $\lambda$ if $\phi_{\beta}=0$ for all $\beta<\lambda$. This first step agrees with the ideas of [46], where, however, the authors do not perform a Chapman-Enskog expansion, but a Maxwellian iteration [35].

In Step 2, new variables are introduced by linear combination of the moments originally chosen. The new variables are constructed such that the number of moments at a given order $\lambda$ is minimal. This step does not only simplify the later discussion, but gives an unambiguous set of moments at order $\lambda$. This ensures that the final result will be independent of the initial choice of moments. Note that, while the basic set of moments (25) makes it easy to identify the order of magnitude (in Step 1), any alternative complete set of moments could have been chosen to arrive at the same new variables after Step 2.

Step 3 follows from the definition of the order of accuracy $\lambda_{0}$ : A set of equations is said to be accurate of order $\lambda_{0}$, when stress $\sigma_{i j}$ and heat flux $q_{i}$ are known within the order $\mathcal{O}\left(\mathrm{Kn}^{\lambda_{0}}\right)$. The evaluation of this condition is based on the fact that all moment equations are strongly coupled. This implies that each term in any of the moment equations has some influence on all other equations, in particular on the conservation laws. A theory of order $\lambda_{0}$ will consider only those terms in all equations whose leading order of influence in the conservation laws is $\lambda \leq \lambda_{0}$. Luckily, in order to evaluate this condition, it suffices to start with the conservation laws, and step by step, order by order, add the relevant terms that are required

The accounting for the order of accuracy is the main difference between the order of magnitude approach and Consistently Ordered Extended Thermodynamics (COET) [46], which assumes that all terms in all moment equations that are of leading order $\lambda \leq \lambda_{0}$ or smaller must be retained. The order of magnitude approach leads to smaller systems of equations for a given order, and can be performed for the full three dimensional and time dependent equations, while [46] presents the equations only for one-dimensional steady state processes.

The order of magnitude method was applied to the special cases of Maxwell molecules and the BGK model in [44, 3], and it was shown that it yields the Euler equations at zeroth order, the Navier-Stokes-Fourier equations at first order, and Grad's 13 moment equations (with omission of the non-linear term $\frac{\sigma_{i j}}{\rho} \frac{\partial \sigma_{j k}}{\partial x_{k}}$ ) at second order. The regularized 13 moment equations (R13) are obtained as the third order approximation, they consists of the conservation laws (7) and the balance laws for stress (19) and heat flux (20) which now are closed by the expressions ${ }^{\dagger}$

$$
\begin{aligned}
\Delta & =-\frac{\sigma_{i j} \sigma_{i j}}{\rho}-12 \frac{\mu}{p}\left[\theta \frac{\partial q_{k}}{\partial x_{k}}+\theta \sigma_{k l} \frac{\partial v_{k}}{\partial x_{l}}+\frac{5}{2} q_{k} \frac{\partial \theta}{\partial x_{k}}-q_{k} \theta \frac{\partial \ln \rho}{\partial x_{k}}\right] \\
R_{i j} & =-\frac{4}{7} \frac{1}{\rho} \sigma_{k\langle i} \sigma_{j\rangle k}-\frac{24}{5} \frac{\mu}{p}\left[\theta \frac{\partial q_{\langle i}}{\partial x_{j\rangle}}+q_{\langle i} \frac{\partial \theta}{\partial x_{j\rangle}}-\theta q_{\langle i} \frac{\partial \ln \rho}{\partial x_{j\rangle}}+\frac{10}{7} \theta \sigma_{k\langle i} \frac{\partial v_{\langle j\rangle}}{\partial x_{k\rangle}}\right] \\
m_{i j k} & =-2 \frac{\mu}{p}\left[\theta \frac{\partial \sigma_{\langle i j}}{\partial x_{k\rangle}}-\sigma_{\langle i j} \theta \frac{\partial \ln \rho}{\left.\partial x_{k}\right\rangle}+\frac{4}{5} q_{\langle i} \frac{\partial v_{j}}{\partial x_{k\rangle}}\right] .
\end{aligned}
$$

\footnotetext{
$\dagger$ There are some differences between the original R13 equations of [13] and the equations presented here, which result from the order of magnitude method. The original equations contain some higher (4th) order terms, and were derived for the linearized collision operator, see [3] for details and discussion.
} 
The moments of the collision operator $(24)_{2,3}$ are exact for Maxwell molecules, and remain unchanged, $\mathcal{P}_{i j}=-\frac{p}{\mu} \sigma_{i j}, \mathcal{P}_{i}=-\frac{2}{3} \frac{p}{\mu} q_{i}$.

A closer inspection of the regularized equations (26) shows that the terms added to the original Grad 13 moment equations are of super-Burnett order.

For general, i.e. non-Maxwellian, molecule types the order of magnitude method was performed to second order in $[45,3]$; the derivation of the third order equations would be far more involved than for Maxwell molecules. Again the equations at zeroth and first order are the Euler and NSF equations (with exact viscosity, heat conductivity and Prandtl number). The second order equations are a generalization of Grad's 13 moment equations, ${ }^{\dagger}$

$$
\begin{aligned}
& \frac{D \sigma_{i j}}{D t}+\sigma_{i j} \frac{\partial v_{k}}{\partial x_{k}}+2 \sigma_{k\langle i} \frac{\partial v_{j\rangle}}{\partial x_{k}}+\frac{4}{5} \operatorname{Pr} \frac{\varpi_{3}}{\varpi_{2}}\left(\frac{\partial q_{\langle i}}{\partial x_{j\rangle}}-\omega q_{\langle i} \frac{\partial \ln \theta}{\partial x_{j\rangle}}\right) \\
& +\frac{4}{5} \operatorname{Pr} \frac{\varpi_{4}}{\varpi_{2}} q_{\langle i} \frac{\partial \ln p}{\partial x_{j\rangle}}+\frac{4}{5} \operatorname{Pr} \frac{\varpi_{5}}{\varpi_{2}} q_{\langle i} \frac{\partial \ln \theta}{\partial x_{j\rangle}}+\left(\frac{\varpi_{6}}{\varpi_{2}}-4\right) \sigma_{k\langle i} S_{j\rangle k} \\
& =-\frac{2}{\varpi_{2}} \frac{p}{\mu}\left[\sigma_{i j}+2 \mu \frac{\partial v_{\langle i}}{\partial x_{j\rangle}}\right], \\
& \frac{D q_{i}}{D t}+q_{k} \frac{\partial v_{i}}{\partial x_{k}}+\frac{5}{3} q_{i} \frac{\partial v_{k}}{\partial x_{k}}-\frac{5}{2} \frac{1}{\operatorname{Pr}} \sigma_{i k} \frac{\partial \theta}{\partial x_{k}}+\frac{5}{4} \frac{1}{\operatorname{Pr}} \frac{\theta_{3}}{\theta_{2}} \theta \sigma_{i k} \frac{\partial \ln p}{\partial x_{k}} \\
& +\frac{5}{4} \frac{1}{\operatorname{Pr}} \frac{\theta_{4}}{\theta_{2}} \theta\left(\frac{\partial \sigma_{i k}}{\partial x_{k}}-\omega \sigma_{i k} \frac{\partial \ln \theta}{\partial x_{k}}\right)+\frac{5}{2} \frac{1}{\operatorname{Pr}} \frac{3}{2} \frac{\theta_{5}}{\theta_{2}} \sigma_{i k} \frac{\partial \theta}{\partial x_{k}} \\
& =-\frac{1}{\theta_{2}} \frac{5}{2} \frac{1}{\operatorname{Pr}} \frac{p}{\mu}\left[q_{i}+\frac{5}{2} \frac{\mu}{\operatorname{Pr}} \frac{\partial \theta}{\partial x_{i}}\right] .
\end{aligned}
$$

Here, the coefficients $\varpi_{\alpha}, \theta_{\alpha}$ are the Burnett coefficients of Table 1 and $\omega$ is the viscosity exponent of (15).

Jin and Slemrod $[47,48]$ proposed an alternative regularization by constructing a set of equations that (a) gives the Burnett equations in a second order CE expansion, and (b) gives a positive entropy production for all values of the variables. Up to second order their equations agree with the generalized Grad 13 equations $(27,28)$ to which they add terms of super-Burnett order that were designed to achieve their goal (b). These higher order terms cannot be justified from the Boltzmann equation $[3]$.

We summarize as follows: The order of magnitude method reproduces the established results of the CE expansion at zeroth (Euler) and first (NSF) order. Moreover it provides a new link between the Knudsen number and Grad's 13 moment equations which turn out to be of second order in the Knudsen number, together with a generalization of these for non-Maxwellian molecules. Finally, the method provides a rational derivation of the R13 equations that does not require artificial assumptions.

\section{Relations between the various sets of equations}

The derivation of macroscopic equations from the Boltzmann equation is simplest for the special case of Maxwell molecules. Accordingly, theories of higher orders

\footnotetext{
† Recall that Grad's 13 moment equations are only suitable for Maxwell molecules.
} 


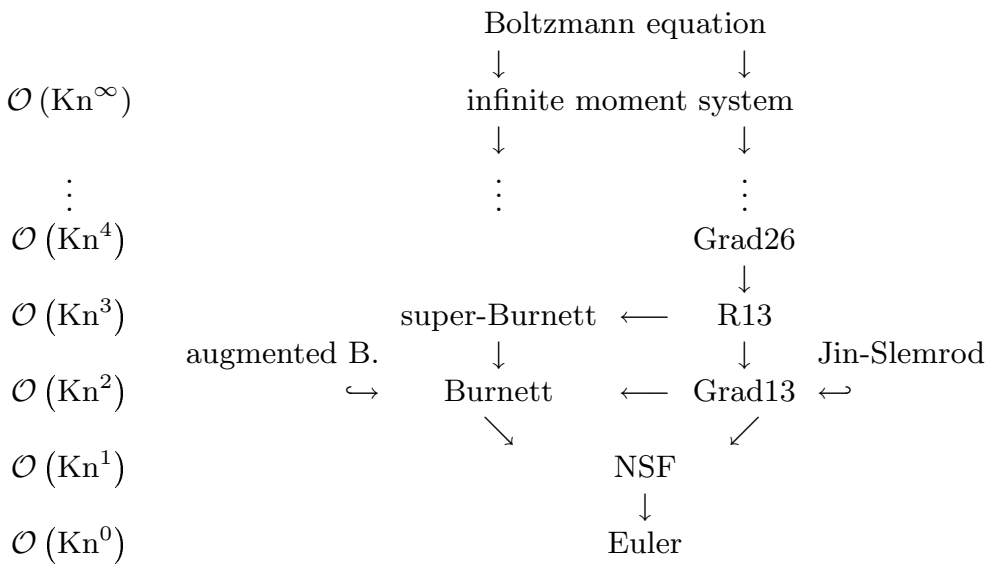

Table 2: The hierarchy of macroscopic equations for Maxwell molecules [3].

in the Knudsen number, like the super-Burnett and the R13 equations, are - at present - only available for Maxwell molecules.

The Chapman-Enskog expansion of increasing order gives the Euler, NavierStokes-Fourier, Burnett, and super-Burnett equations.

The augmented Burnett equations contain terms of super-Burnett order, which are added ad hoc, and cannot be derived from the Boltzmann equation.

Grad-type moment equations can be constructed for arbitrary moment sets, but the 13 moment system and the 26 moment system are particularly interesting, since they are equations of orders $\mathcal{O}\left(\mathrm{Kn}^{2}\right)$ and $\mathcal{O}\left(\mathrm{Kn}^{4}\right)$, respectively. They can be obtained also from the order of magnitude approach which gives the R13 equations as the proper equations at order $\mathcal{O}\left(\mathrm{Kn}^{3}\right)$, and the NSF and Euler equations at orders $\mathcal{O}\left(\mathrm{Kn}^{1}\right)$ and $\mathcal{O}\left(\mathrm{Kn}^{0}\right)$, respectively.

Jin and Slemrod's equations are accurate to order $\mathcal{O}\left(\mathrm{Kn}^{2}\right)$, but contain terms of super-Burnett order, $\mathcal{O}\left(\mathrm{Kn}^{3}\right)$, which cannot be derived from the Boltzmann equation.

A Chapman-Enskog expansion of higher order moment equations can be performed by means of CE expansions for stress and heat flux (see (13)); the results agree with those of the CE expansion of the Boltzmann equation.

The relations between the various sets of equations are depicted in Table 2, in which an arrow between two sets of equations indicates that one set can be derived from the other (e.g. the Burnett eqs. from the Grad13 eqs. by means of a CE expansion). Note that at a given order the equations derived from the CE method and from the order of magnitude approach are quite different, due to the marked differences in methodology. Indeed, the CE based equations (e.g. the super-Burnett equations, at third order), contain less information than their counterparts (e.g. the R13 equations, also at third order), since the former can be derived from the latter, but not vice versa.

For other types of interaction potentials, accurate sets of equations are only available to order $\mathcal{O}\left(\varepsilon^{2}\right)$, namely the generalized 13 moment equations which were 


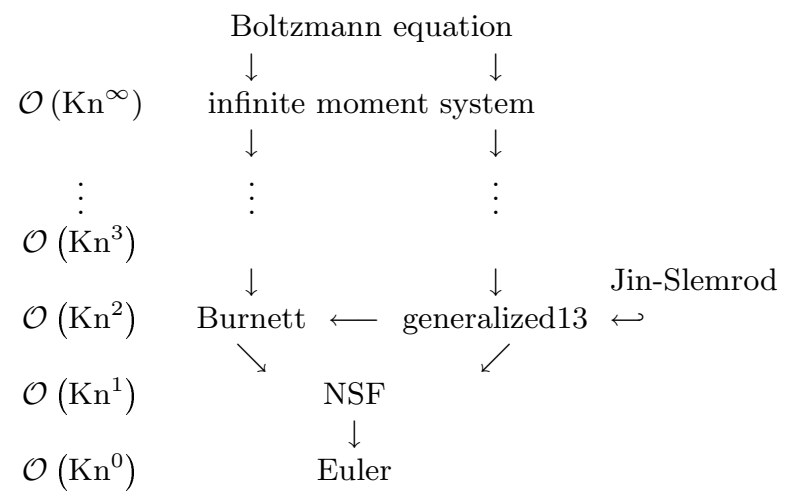

Table 3: The hierarchy of macroscopic equations for molecules with arbitrary interaction potentials [3].

obtained from the order of magnitude method, and the Burnett equations from the CE method. The Euler and NSF equations form the proper equations at zeroth and first order. The Jin-Slemrod equations are available as well. Table 3 shows the known equations as well as their order of accuracy, and their relations.

\section{Applications}

The previous sections gave an overview over several methods to derive macroscopic equations for rarefied gas flows; see Tables 2 and 3 . We now turn the attention to some applications in order to discuss the behavior and quality of the equations. As before, space restrictions forbid to go into detail, and the interested reader is referred to the cited literature.

Linear stability: Bobylev [12] has shown that the Burnett and super-Burnett equations are unstable in transient problems. This failure is the most important reason to discard the Burnett and super-Burnett equations (and thus the CE expansion), and to strive for alternative methods.

For one-dimensional processes the linearized transport equations of the previous sections can be written as

$$
\frac{\partial u_{A}}{\partial t}+\mathcal{A}_{A B} \frac{\partial u_{B}}{\partial x}+\mathcal{B}_{A B} \frac{\partial^{2} u_{B}}{\partial x^{2}}+\cdots=\mathcal{C}_{A B} u_{B}
$$

with constant matrices $\mathcal{A}_{A B}, \mathcal{B}_{A B}, \mathcal{C}_{A B}, \ldots$ For the solution, we assume plane waves of the form

$$
u_{A}=\tilde{u}_{A} \exp [i(\Omega t-k x)]
$$

where $\tilde{u}_{A}$ is the complex amplitude of the wave, $\Omega$ is its frequency, and $k$ is its wave number. The equations (29) can then be written as

$$
\mathcal{G}_{A B}(\Omega, k) \tilde{u}_{B}=0 \text { where } \mathcal{G}_{A B}(\Omega, k)=i \Omega \delta_{A B}-i k \mathcal{A}_{A B}-k^{2} \mathcal{B}_{A B}+\cdots-\mathcal{C}_{A B}
$$


and nontrivial solutions require $\operatorname{det}\left[\mathcal{G}_{A B}(\Omega, k)\right]=0$; the resulting relation between $\Omega$ and $k$ is the dispersion relation.

If a disturbance in space is considered, the wave number $k$ is real, the frequency is complex, $\Omega=\Omega_{r}(k)+i \Omega_{i}(k)$, and stability requires $\Omega_{i}(k) \geq 0$.

If a disturbance in time at a given location is considered, the frequency $\Omega$ is real, while the wave number is complex, $k=k_{r}(\Omega)+i k_{i}(\Omega)$. Then, for a wave traveling in positive $x$-direction $\left(k_{r}>0\right)$, the damping must be negative $\left(k_{i}<0\right)$, while for a wave traveling in negative $x$-direction $\left(k_{r}<0\right)$, the damping must be positive $\left(k_{i}>0\right)$. Thus, if $k(\Omega)$ is plotted in the complex plane with $\Omega$ as parameter, the curves should not touch the upper right nor the lower left quadrant.

Thus, in order to test the stability of a given set of equations, one has to test for stability in time and space. However, for the Burnett and super-Burnett equations, most authors only consider stability in time, and ignore stability in space $[12,19]$.
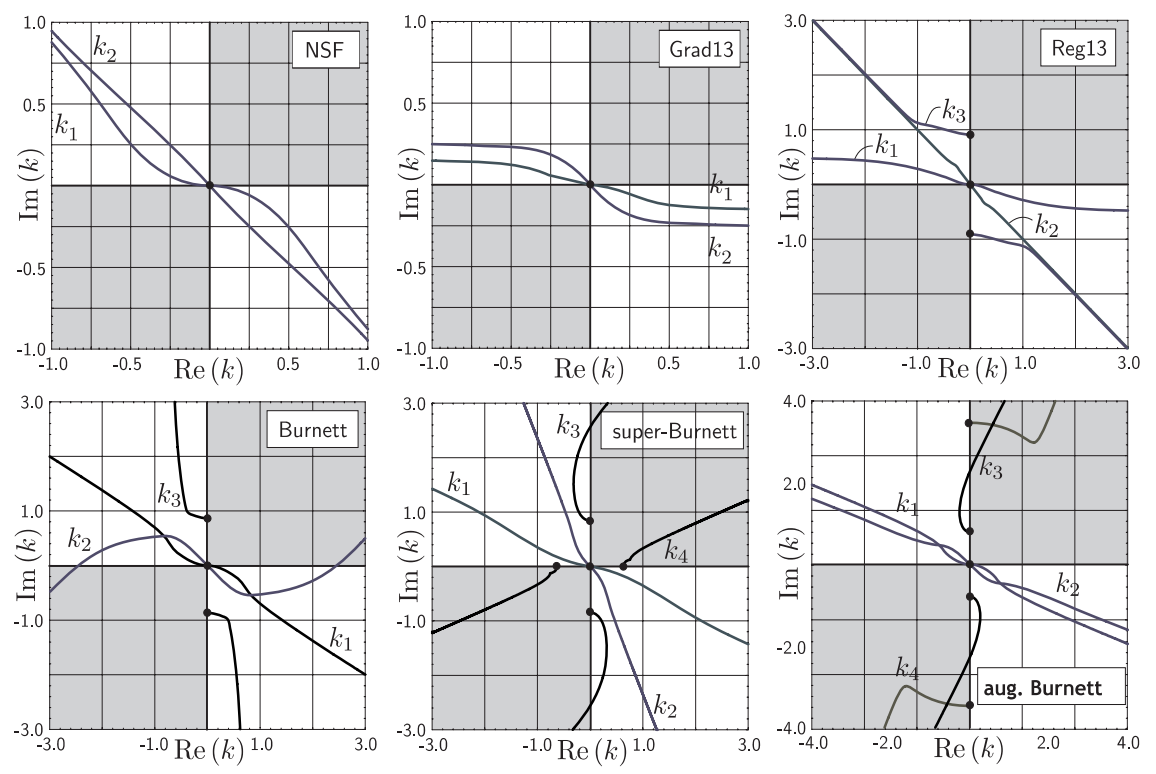

Fig. 1: The solutions $k(\Omega)$ of the dispersion relation in the complex plane with $\Omega$ as parameter for Navier-Stokes-Fourier, Burnett, Super-Burnett, augmented Burnett, Grad 13, and R13 equations [13, 15]. The dots denote the points where $\Omega=0$.

Figure 1 , taken from $[13,15]$, considers the stability against local disturbances of frequency $\Omega$. The figure shows the solutions of the dispersion relation for the different sets of equations; the dots mark the points where $\Omega=0$. Grad 13 equations, and NSF equations give two different modes each, and none of the solutions violates the condition of stability. The R13 equations have 3 modes, all of them are stable. This is different for the Burnett (3 modes), super-Burnett (4 modes), and augmented Burnett (4 modes) equations: the Burnett equations have one unstable mode, and super-Burnett and augmented Burnett have two unstable modes. 
The test for stability in time [15] shows that NSF, augmented Burnett, Grad 13, and R13 equations are stable for all wavelengths, while Burnett and super-Burnett equations are unstable.

All Burnett type equations, including the augmented Burnett equations, fail the tests for stability. The NSF, Grad-type equations and the R13 equations are stable for all frequencies and for disturbances of any wavelength.

Shock structures: The computation of shock structures is a standard test for macroscopic equations designed to describe rarefied gas flows, and we present some of the results of [15].

A one-dimensional steady shock structure connects two equilibrium states, where the values of density $\rho_{0}, \rho_{1}$, velocity $v_{0}, v_{1}$, and temperature $\theta_{0}, \theta_{1}$ in the two equilibrium states are related through the Rankine-Hugoniot relations [3]. The relevant parameter for the shock is the inflow Mach number $M_{0}=v_{0} / \sqrt{\frac{5}{3} \theta_{0}}$.

We compare the shock structures obtained from macroscopic equations for rarefied flows to DSMC results obtained with Bird's code [5], and plot results in dimensionless form [51, 15, 3]. Figure 2 shows the density and heat flux profiles of a $M_{0}=2$ shock calculated with the NSF and Grad13 equations as well as with the Burnett and super-Burnett equations for Maxwell molecules. The NSF result simply mismatches the profile, while the Grad13 solution shows a strong subshock. The Burnett and super-Burnett solutions are spoiled by oscillations in the downstream part of the shock, which arise due to the spatial instabilities. Thus, for the computation of shock structures the Burnett equations and super-Burnett-equations have to be rejected.
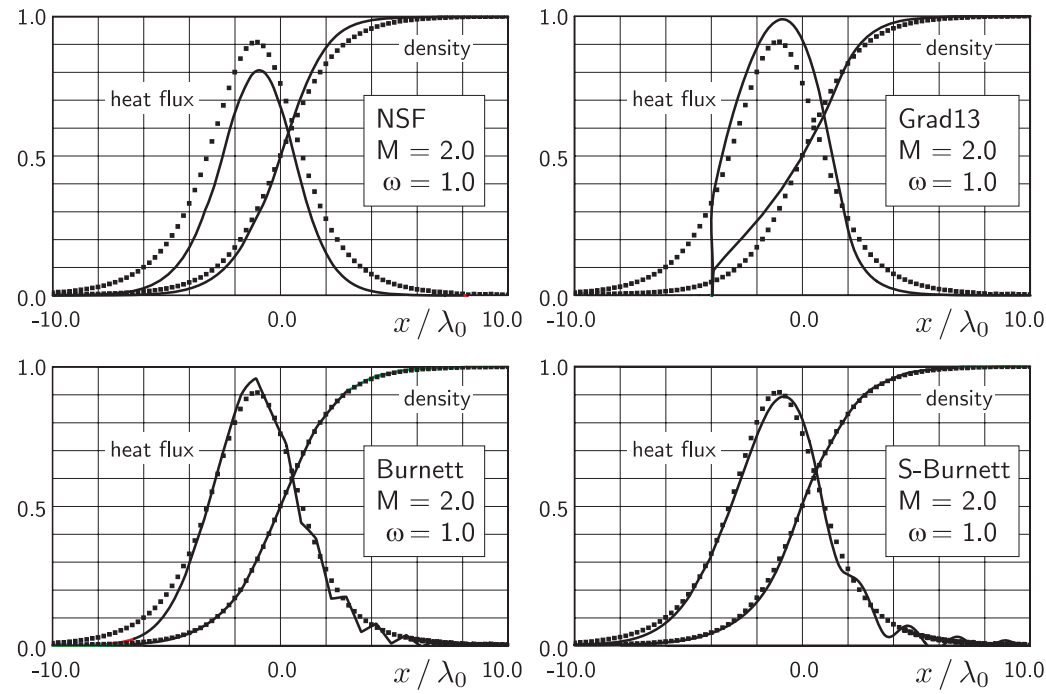

Fig. 2: Shock structure solutions for the NSF equations, Grad 13 equations, and Burnett and super-Burnett equations, for Maxwell molecules at Mach number $M_{0}=2$ (solid lines). Both Burnett results exhibit non-physical oscillations in the downstream region. The squares represent the DSMC solution. 

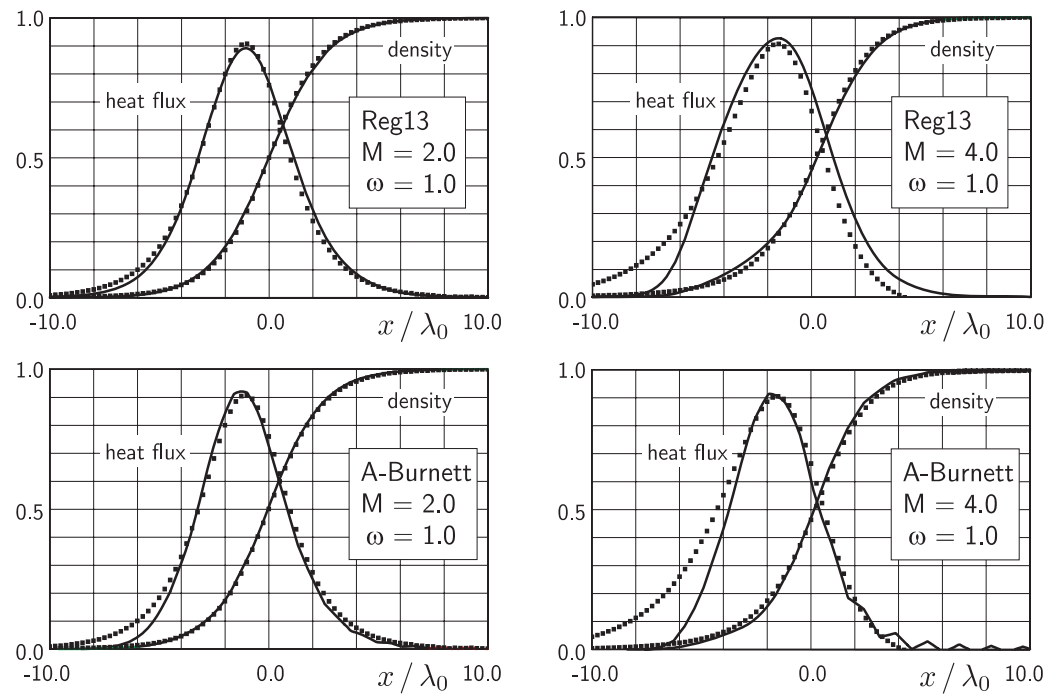

Fig. 3: Shock structures in a gas of Maxwell molecules with Mach numbers $M_{0}=2$ and $M_{0}=4$. The upper row shows the solution of the R13 equations, while the lower row shows the results of the augmented Burnett equations. The squares correspond to the DSMC solution.

In [52] DSMC results for velocity and temperature are used to compute stress $\sigma$ and heat flux $q$ from the Burnett equations. Comparison with the actual DSMC results for $\sigma$ and $q$ shows considerable improvement over the NSF equations. Thus, the Burnett equations contain the proper physics of the shock, but are useless, since their mathematical structure does not allow to compute a stable solution. Fiscko and Chapman [14] deleted one linear term from the Burnett and super-Burnett equations to obtain stable shock solutions in reasonable agreement to DSMC simulations. Obviously, the mathematical properties of the equations are changed by deleting terms ad hoc, and thus it is not surprising that they obtained stable behavior.

R13 equations and augmented Burnett equations give good results for a wider range of Mach numbers. Figure 3 compares shock structures for Maxwell molecules at $M_{0}=2$ and $M_{0}=4$ for R13 and augmented Burnett with DSMC results. For $M_{0}=2$ the density profiles exhibit no visible differences and both models match the DSMC results very well. The shape of the heat flux is captured very well by the R13 equations, while the augmented Burnett equations do not reproduce the maximum value and the upstream relaxation. The deviations from the DSMC solutions are more pronounced for $M_{0}=4$, where the R13 results begin to deviate in the upstream part. In the tail of the augmented Burnett profiles small oscillations are present, due to instability in space. This happens since the solution was obtained from a boundary value problem; no stability problems arise when the augmented Burnett equations are solved by by time stepping into steady state [19, 20]. Altogether, the results of the R13 system for Maxwell molecules agree better with DSMC results than the solutions of the augmented Burnett equations. For higher Mach numbers both deviate somewhat from DSMC results. 
A shock is often characterized by the shock thickness, defined as $[49,50]$

$$
\delta=\frac{\rho_{1}-\rho_{0}}{\max \left(\frac{\partial \rho}{\partial x}\right)} .
$$

Figure 4 compares thickness results for the R13 system to measurements in argon $(\omega=0.8)[49,50]$. The computed shock thickness yields a striking agreement with the experimental data. The results of the augmented Burnett equations with $\omega=0.8$ lead to a similar agreement, while the NSF results lie far off. The good agreement of the shock thickness for high Mach numbers should not be overemphasized, since the single parameter $\delta$ cannot reflect the complete profile, so that the agreement with shock thickness measurements does not imply a reliable description of the complete profile. Nevertheless, the information that $\delta$ does reflect - a mean thickness - is predicted by the R13 equations accurately even for high Mach numbers.

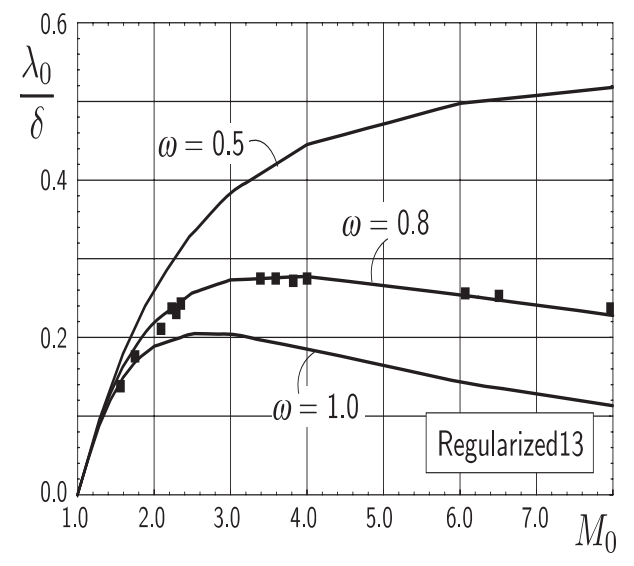

Fig. 4: Comparison of the inverse shock thickness for the R13 equations with measurements for Argon (squares, $\omega \approx 0.8$ ). The curve of the augmented Burnett equations with $\omega=0.8$ shows a similar agreement.

Couette flow: The biggest obstacle for any higher order model for rarefied gas flows is to find proper boundary conditions. This is a difficult problem, and no conclusive answers can be given at present. We shall not discuss the problems, but only present some Couette flow calculations that were obtained by partial fitting of boundary conditions. Couette flow describes the motion of a gas between two infinite parallel plates at distance $L$, moving against each other with the speed $v_{W}^{L}$, details can be found in [3].

For constructing the solution it is assumed that the non-equilibrium quantities can be split into bulk (B) and Knudsen layer (L) contributions, $\phi=\phi_{B}+\phi_{L}$, where the Knudsen layer contributions vanish in some distance from the wall.

The bulk equations follow from a Chapman-Enskog expansion of the steady state equations in Couette geometry; only the second order equations are considered, which read ( $y=x_{2} / L$ is the dimensionless space variable) 


$$
\sigma_{12}=-\frac{\mu P_{0}}{\rho \theta L} \frac{d v}{d y}, q_{2}=-\frac{15}{4} \frac{\mu P_{0}}{\rho \theta L} \frac{d \theta}{d y}, \sigma_{22}=-\frac{6}{5} \frac{\sigma_{12} \sigma_{12}}{P_{0}}, q_{1}=\frac{7}{2} \frac{\sigma_{12} q_{2}}{P_{0}} .
$$

The first two equations, for $\sigma_{12}$ and $q_{2}$, are the laws of Navier-Stokes and Fourier multiplied with the factor $P_{0} / \rho \theta=P_{0} / p$. When these are used with the conservation laws (7), it suffices to prescribe the jump and slip boundary conditions [3]

$$
\begin{aligned}
& v^{\alpha}-v_{W}^{\alpha}=\frac{-\frac{2-\chi}{\chi} \alpha_{1} \sqrt{\frac{\pi}{2}} \sqrt{\theta} \sigma_{12} n^{\alpha}-\frac{1}{5} \alpha_{2} q_{1}}{\rho \theta+\frac{1}{2} \sigma_{22}}, \\
& \theta^{\alpha}-\theta_{W}^{\alpha}=-\frac{\frac{2-\chi}{2 \chi} \beta_{1} \sqrt{\frac{\pi}{2}} \sqrt{\theta} q_{2} n^{\alpha}+\frac{1}{4} \theta \sigma_{22}}{\rho \theta+\frac{1}{2} \sigma_{22}}+\frac{V^{2}}{4},
\end{aligned}
$$

with correction factors $\alpha_{1}, \alpha_{2}, \beta_{1}$ close to unity [1,3]. The constant $P_{0}$ follows from the prescribed mass between the plates.

More interesting are the equations for the normal stress, $\sigma_{22}$, and the heat flux parallel to the wall, $q_{1}$. Both vanish in the NSF theory, and thus their non-zero values describe pure rarefaction effects. In particular it must be noted that there is no temperature gradient in the $x$-direction: $q_{1}$ is a heat flux that is not driven by a temperature gradient.

Depending on the set of equations used, $\sigma_{i j}$ and $q_{i}$ can have linear Knudsen layer contributions as well. The CE expansion, that gave the bulk solution, discards these linear parts [3], but they can be obtained from the linearized equations [16].

The superposition of bulk solution and Knudsen layers for the R13 equations gives

$$
\begin{aligned}
& v=v_{\mid B}-\frac{2}{5} q_{1 \mid L}, \quad \theta=\theta_{\mid B}-\frac{2}{5} \sigma_{22 \mid L}, \quad \sigma_{12}=\sigma_{12 \mid B}, \quad \sigma_{22}=\sigma_{22 \mid B}+\sigma_{22 \mid L} \\
& p=P_{0}-\sigma_{22 \mid L}, \quad \rho=\frac{p}{\theta}, \quad q_{1}=q_{1 \mid B}+q_{1 \mid L}, \quad q_{2}=q_{2 \mid B} .
\end{aligned}
$$

with the Knudsen layer terms

$$
q_{1 \mid L}=A \sinh \left[\sqrt{\frac{5}{9}} \frac{y-\frac{1}{2}}{\mathrm{Kn}}\right] \quad, \quad \sigma_{22 \mid L}=D \cosh \left[\sqrt{\frac{5}{6}} \frac{y-\frac{1}{2}}{\mathrm{Kn}}\right] .
$$

The constants of integration $A$ and $D$, which should be computed from boundary conditions for stress and heat flux, were fitted to DSMC simulations. Figure 5 compares results of DSMC calculations, NSF equations with jump and slip boundary conditions, and the $\mathrm{R} 13$ equations for $\mathrm{Kn}=0.1$. R13 matches the DSMC simulations quite well; the most visible differences lie in the bulk values for $\sigma_{12}$ and $\sigma_{22}$. The temperature maximum is reproduced very well, while some differences can be observed at the boundaries. NSF, on the other hand, cannot neither describe Knudsen boundary layers nor the rarefaction effects described by $\sigma_{22}$ and $q_{1}$.

NSF and Grad 13 equations do not give linear Knudsen layers at all. The Burnett and super-Burnett equations cannot describe Knudsen layers of the type (34) but give periodic solutions of the form $A \sin \left[\lambda \frac{x-1 / 2}{\mathrm{Kn}}\right], B \cos \left[\lambda \frac{x-1 / 2}{\mathrm{Kn}}\right]$. The augmented Burnett equations give expressions of the type (34), but the signs for the heat flux parallel to the flow does not match the DSMC simulations [16]. 

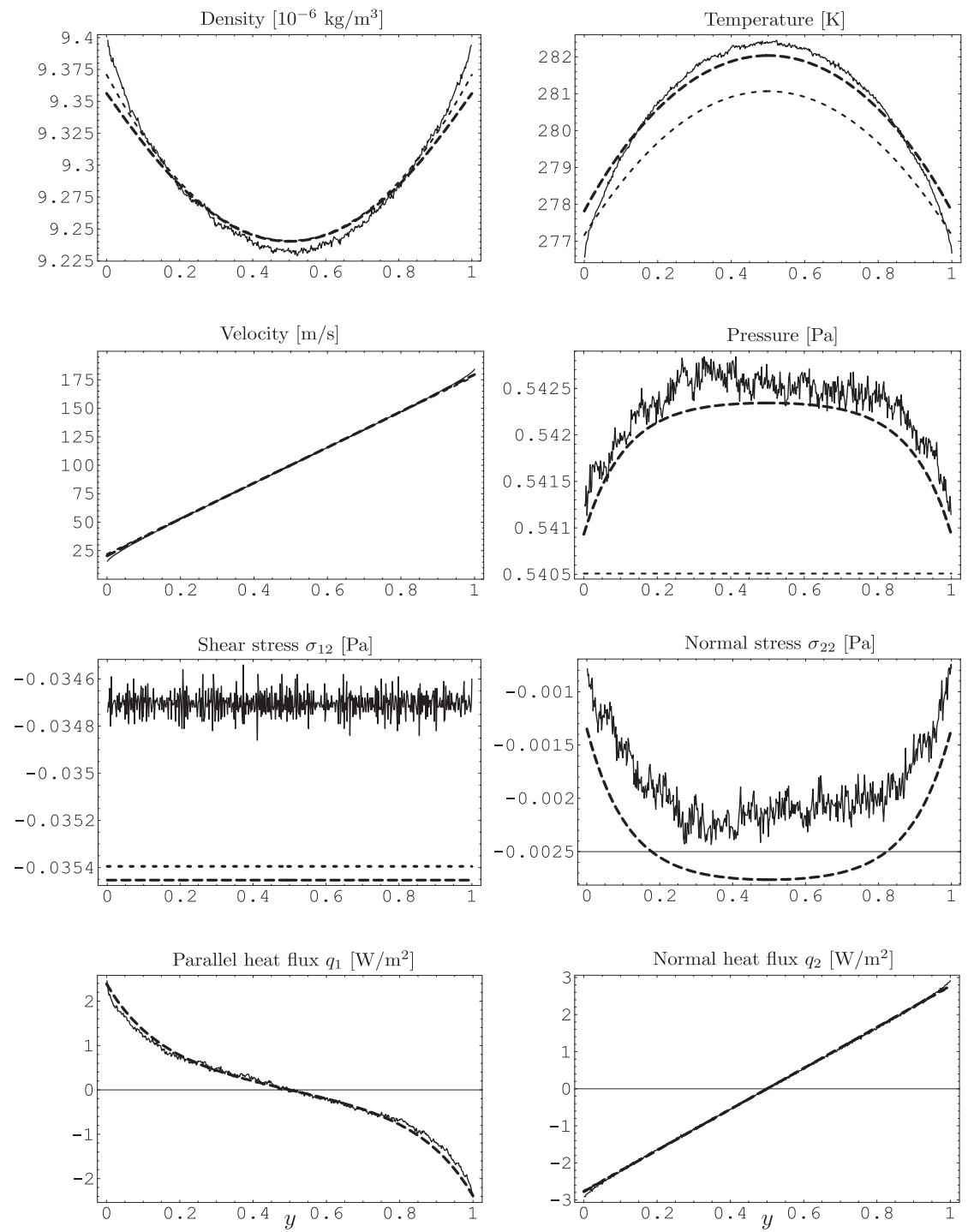

Fig. 5: Couette flow at $\mathrm{Kn}=0.1$, with $v_{W}^{L}=200 \frac{\mathrm{m}}{\mathrm{s}}$. Continuous line: DSMC, finely dashed line (- - - ): NSF, dashed line (-- - ): superposition of bulk solution and linear Knudsen layer solution. Recall that NSF implies $q_{1}=\sigma_{22}=0$ (curves not shown). 


\section{Conclusions and Outlook}

Several methods to derive macroscopic equations for rarefied gases, and the resulting equations were presented, including the classical Chapman-Enskog and Grad methods, and the new order of magnitude method. Our interest was focussed on equations for Knudsen numbers above 0.01, i.e. beyond the validity of the NavierStokes equations.

The CE method suggests the Burnett and super-Burnett equations which, however, are unstable, lead to spurious oscillations in linear steady state processes, and fail to accurately describe shock structures. Efforts to augment the equations were only partially successful.

The Grad method leads to stable equations that can describe Knudsen layers when more than 13 variables are considered. Due to the hyperbolicity of the equations, they lead to unphysical subshocks in shock structure problems.

The order of magnitude method suggest the Grad 13 moment equations at second order and the R13 equations at third order. The latter are superior to the competing sets of equations for several reasons: (a) they contain the Burnett and Super-Burnett equations as can be seen by means of a CE expansion in the Knudsen number, (b) they are linearly stable for all wavelengths and frequencies, (c) they show phase speeds and damping coefficients that match experiments better than those for the NSF equations, or the Grad13 system, (d) they exhibit Knudsen boundary layers, and (e) they lead to smooth shock structures for all Mach numbers.

While the R13 equations have many desirable features, a number of difficult problems must be solved before the R13 equations (or any other model above the NSF equations) can be used as a reliable tool. (a) Reliable boundary conditions must be developed. (b) Industrially relevant gases are diatomic (air!) or polyatomic, and higher order equations for these and for mixtures must be derived. (c) The multiscale character of rarefied flows requires advanced numerical methods that, based on a well chosen local Knudsen number, use the most efficient set of equations in a flow region; this requires the interplay of solvers for NSF, R13 and Boltzmann equations, and reliable switching and transition conditions. (d) Currently, only the Jin-Slemrod equations are accompanied by a proper entropy inequality, and it is desirable to find equivalents for the other higher order models.

These problems are under investigation, and we hope to be able to present solutions in the future.

Acknowledgement. This research was supported by the Natural Sciences and Engineering Research Council (NSERC).

\section{References}

1. C. Cercignani: Theory and application of the Boltzmann Equation. Scottish Academic Press, Edinburgh 1975

2. S. Chapman, T.G. Cowling: The mathematical Theory of Non-Uniform Gases (Cambridge University Press 1970)

3. H. Struchtrup: Macroscopic Transport Equations for Rarefied Gas FlowsApproximation Methods in Kinetic Theory, Interaction of Mechanics and Mathematics Series (Springer, Heidelberg 2005) 
4. T. Ohwada: Heat flow and temperature and density distributions in a rarefied gas between parallel plates with different temperatures. Finite difference analysis of the nonlinear Boltzmann equation for hard sphere molecules. Phys. Fluids $\mathbf{8}$, 2153-2160 (1996)

5. G. Bird: Molecular gas dynamics and the direct simulation of gas flows (Clarendon Press, Oxford 1994)

6. P.L. Bhatnagar, E.P. Gross, M. Krook: A Model for collision processes in gases. I. Small Amplitude Processes in Charged and Neutral One-Component Systems. Phys. Rev. 94, 511-525 (1954)

7. M.N. Kogan: Rarefied Gas Dynamics (Plenum Press, New York 1969)

8. J.H. Ferziger, H.G. Kaper: Mathematical theory of transport processes in gases (North-Holland, Amsterdam 1972)

9. D. Burnett: The distribution of molecular velocities and the mean motion in a non-uniform gas. Proc. Lond. Math. Soc. 40, 382-435 (1936)

10. S. Reinecke, G.M. Kremer: Method of Moments of Grad. Phys. Rev. A 42, 815-820 (1990)

11. M.Sh. Shavaliyev: Super-Burnett Corrections to the Stress Tensor and the Heat Flux in a Gas of Maxwellian Molecules. J. Appl. Maths. Mechs. 57, 573-576 (1993)

12. A.V. Bobylev: The Chapman-Enskog and Grad methods for solving the Boltzmann equation. Sov. Phys. Dokl. 27, 29-31 (1982)

13. H. Struchtrup, M. Torrilhon: Regularization of Grad's 13-moment-equations: Derivation and Linear Analysis. Phys. Fluids 15, 2668-2680 (2003)

14. K.A. Fiscko, D.R. Chapman: Comparison of Burnett, Super-Burnett and Monte Carlo Solutions for Hypersonic Shock Structure. In: Proceedings of the 16th Symposium on Rarefied Gasdynamics (AIAA, Washington 1989), 374-395

15. M. Torrilhon, H. Struchtrup: Regularized 13-Moment-Equations: Shock Structure Calculations and Comparison to Burnett Models. J. Fluid Mech. 513, 171198 (2004)

16. H. Struchtrup: Failures of the Burnett and Super-Burnett equations in steady state processes. Cont. Mech. Thermodyn. 17, 43-50 (2005)

17. I.V. Karlin, A.N. Gorban: Hydrodynamics from Grad's equations: What can we learn from exact solutions? Ann. Phys. - Berlin 11, 783-833 (2002)

18. Y. Zheng, H. Struchtrup: Burnett equations for the ellipsoidal statistical BGK Model. Cont. Mech. Thermodyn. 16, 97-108 (2004)

19. X. Zhong, R.W. MacCormack, D.R. Chapman: Stabilization of the Burnett Equations and Applications to High-Altitude Hypersonic Flows. AIAA 91-0770 (1991)

20. X. Zhong, R.W. MacCormack, D.R. Chapman: Stabilization of the Burnett Equations and Applications to Hypersonic Flows. AIAA Journal 31, 1036 (1993)

21. H. Grad: On the Kinetic Theory of Rarefied Gases. Comm. Pure Appl. Math. 2, 325 (1949)

22. H. Grad: Principles of the Kinetic Theory of Gases. In: Handbuch der Physik, vol. 12, ed. by S. Flügge (Springer, Berlin 1958)

23. I. Müller, T. Ruggeri: Rational Extended Thermodynamics, Springer Tracts in Natural Philosophy, vol. 37 (Springer, New York 1998)

24. H. Struchtrup: Heat Transfer in the Transition Regime: Solution of Boundary Value Problems for Grad's Moment Equations via Kinetic Schemes. Phys. Rev. E 65, 041204 (2002) 
25. H. Struchtrup: An Extended Moment Method in Radiative Transfer: The Matrices of Mean Absorption and Scattering Coefficients. Ann. Phys. 257, 111-135 (1997)

26. W. Weiss: Continuous shock structure in extended Thermodynamics. Phys. Rev. E 52, 5760 (1995)

27. J.D. Au: Nichtlineare Probleme und Lösungen in der Erweiterten Thermodynamik. Dissertation, Technical University Berlin 2000

28. J.D. Au, M. Torrilhon, W. Weiss: The Shock Tube Study in Extended Thermodynamics, Phys. Fluids 13, 2423-2432, (2001)

29. H. Struchtrup: Kinetic schemes and boundary conditions for moment equations. ZAMP 51, 346-365 (2000)

30. H. Struchtrup: Some remarks on the equations of Burnett and Grad. IMA Volume 135 "Transport in Transition Regimes," (Springer, New York 2003)

31. D. Reitebuch, W. Weiss: Application of High Moment Theory to the Plane Couette Flow. Cont. Mech. Thermodyn. 11, 227 (1999)

32. H. Struchtrup: Grad's Moment Equations for Microscale Flows. In: Symposium on Rarefied Gasdynamics 23, AIP Conference Proceedings 663, 792-799 (2003)

33. W. Weiss: Zur Hierarchie der Erweiterten Thermodynamik. Ph.D. thesis, Technical University Berlin (1990)

34. E. Ikenberry, C. Truesdell: On the pressures and the flux of energy in a gas according to Maxwell's kinetic theory I. J. of Rat. Mech. Anal. 5, 1-54 (1956)

35. C. Truesdell, R.G. Muncaster: Fundamentals of Maxwell's kinetic theory of a simple monatomic gas (Academic Press, New Yorck 1980)

36. S. Reinecke, G.M. Kremer: Burnett's equations from a $(13+9 \mathrm{~N})$-field theory. Cont Mech. Thermodyn. 8, 121-130 (1996)

37. I.V. Karlin, A.N. Gorban, G. Dukek, T.F. Nonnenmacher: Dynamic correction to moment approximations, Phys. Rev. E 57 , 1668-1672 (1998)

38. A.N. Gorban, I.V. Karlin: Invariant Manifolds for Physical and Chemical Kinetics, Lecture Notes in Physics, vol. 660, (Springer, Berlin 2005)

39. W. Dreyer: Maximization of the Entropy in Non-equilibrium. J. Phys. A: Math. Gen. 20, 6505 (1987)

40. C.D. Levermore: Moment Closure Hierarchies for Kinetic Theories. J. Stat. Phys. 83, 1021-1065 (1996)

41. M. Junk: Domain of definition of Levermore's five-moment system. J. Stat. Phys. 93, 1143-1167 (1998)

42. W. Dreyer, M. Junk, M. Kunik: On the approximation of the Fokker-Planck equation by moment systems. Nonlinearity 14, 881-906 (2001)

43. M. Junk: Maximum entropy moment problems and extended Euler equations. Transport in Transition Regimes, IMA Vol. Math. Appl. 135, 189-198 (Springer, New York 2003)

44. H. Struchtrup: Stable transport equations for rarefied gases at high orders in the Knudsen number. Phys. Fluids 16 , 3921-3934 (2004)

45. H. Struchtrup: Derivation of 13 moment equations for rarefied gas flow to second order accuracy for arbitrary interaction potentials. Multiscale Model. Simul. 3, 211-243 (2004)

46. I. Müller, D. Reitebuch, W. Weiss: Extended Thermodynamics - Consistent in Order of Magnitude, Cont. Mech. Thermodyn. 15, 113-146 (2003)

47. S. Jin, M. Slemrod: Regularization of the Burnett equations via relaxation. $J$. Stat. Phys. 103, 1009-1033 (2001) 
48. S. Jin, L. Pareschi, M. Slemrod: A Relaxation Scheme for Solving the Boltzmann Equation Based on the Chapman-Enskog Expansion. Acta Mathematicas Applicatae Sinica (English Series) 18, 37-62 (2002)

49. B. Schmidt: Electron Beam Density Measurements in Shock Waves in Argon. J. Fluid Mech. 39, 361 (1969)

50. H. Alsmeyer: Density Profiles in Argon and Nitrogen Shock Waves Measured by the Absorbtion of an Electron Beam, J. Fluid Mech. 74, 497 (1976)

51. G.C. Pham-Van-Diep, D.A. Erwin, E.P. Muntz, Testing Continuum Descriptions of Low-Mach-Number Shock Structures. J. Fluid Mech. 232, 403 (1991)

52. E. Salomons, M. Mareschal, Usefulness of the Burnett Description of Strong Shock Waves. Phys. Rev. Lett. 69, 269-272 (1992) 